\title{
A comparative study of efficacy and safety of flupirtine versus piroxicam in postoperative pain in patients undergoing lower limb surgery
}

This article was published in the following Dove Press journal: Journal of Pain Research

16 October 2017

Number of times this article has been viewed

\author{
Sowmya Chinnaiyan' \\ Narayana Sarala' \\ Heddur Shanthappa Arun² \\ 'Department of Pharmacology, \\ ${ }^{2}$ Department of Orthopaedics, Sri \\ Devaraj Urs Medical College, Sri \\ Devaraj Urs Academy of Higher \\ Education and Research, Kolar, \\ Karnataka, India
}

Background: Effective control of pain postoperatively is essential in providing enhanced patient care and a cost-effective hospital stay. Though many treatment modalities exist for postoperative pain management in orthopedic surgeries they are often accompanied by adverse effects. This study was carried out to assess the efficacy of flupirtine and piroxicam in postoperative pain reduction using visual analog scale (VAS) score.

Materials and methods: An open-label, parallel group, comparative study was conducted on patients undergoing lower limb orthopedic surgery, randomized into two groups of 38 patients each. They received either flupirtine $100 \mathrm{mg}$ or piroxicam $20 \mathrm{mg} 6$ hours after surgery and then twice daily orally for 5 days. Pain was measured using VAS score, total pain relief score $\left(\right.$ TOTPAR $_{24}$ ), and patient satisfaction score (PSS); the other scales used were behavioral pain assessment scale (BPAS) and functional activity score (FAS). Rescue medication used was tramadol $100 \mathrm{mg}$ intravenously. WHO causality scale was used for assessing adverse effects. Descriptive and inferential statistics were used for assessment of various parameters.

Results: A total of 76 patients with mean \pm standard deviation age of $35.08 \pm 10.3$ years were recruited; 34 in the flupirtine and 37 in the piroxicam groups completed the study. Patients in both groups were comparable in baseline characteristics. Flupirtine and piroxicam reduced VAS score 48 hours postoperatively compared to baseline ( $p=0.006$ and 0.001 ) and piroxicam produced significant reduction in pain at 8,12 , and 120 hours compared to flupirtine $(p=0.028,0.032$, 0.021). TOTPAR 24 and PSS at 24 hours were comparable between the treatments. BPAS scores at 24 hours were reduced significantly in patients receiving either drug $(p=0.001)$. FAS improved at 72 hours in patients receiving piroxicam. Adverse effects were similar with both the medications. Conclusion: Flupirtine and piroxicam reduced pain effectively but the onset of pain relief was earlier with piroxicam.

Keywords: postoperative pain, flupirtine, piroxicam, orthopedic surgeries, postoperative analgesia

\section{Introduction}

Pain has been the most common problem encountered in the immediate postoperative period. Effective control of this pain has become essential as it helps in providing early mobilization, better recovery, and cost-effective hospital stay, especially in patients undergoing orthopedic surgeries. Acute pain if left untreated may lead to chronicity and become persistent which imposes a greater burden on the patient, as treatment modalities for chronic pain is limited and rarely effective. ${ }^{1}$ The existing medications used to relieve pain following orthopedic surgeries are nonsteroidal anti-inflammatory drugs (NSAIDs), opioids, local anesthetics, gabapentinoids, antiepileptics, and steroids, but they are often accompanied by adverse effects. ${ }^{2}$ Hence, the need for further studies in this regard has become necessary.
Correspondence: Narayana Sarala Department of Pharmacology, Sri Devaraj Urs Medical College, Sri Devaraj Urs Academy of Higher Education and Research, Tamaka, Kolar, Karnataka, 563103 , India

$\mathrm{Tel}+9|8| 52243003$ III

$\mathrm{Fax}+918152243006$

Email n_sarala@rediffmail.com 
Piroxicam, an oxicam-derived NSAID, inhibits prostaglandin-mediated pain and inflammation. The advantages of this group of drugs over the other analgesics are decreased sensitization of peripheral receptors, absence of addiction potential, and cognitive impairment. It is used for postoperative pain relief, musculoskeletal disorders, and arthritis.

Flupirtine, a nonopioid, non-NSAID drug, belongs to $\mathrm{K}_{\mathrm{v}} 7$ potassium channel openers, exerting its action by exhibiting antagonism on N-methyl-D-aspartate (NMDA) receptors of glutamate. In addition, it also exerts therapeutic action via $\mathrm{GABA}_{\mathrm{A}}$ ergic receptors. ${ }^{3,4}$ It has a unique analgesic and skeletal muscle relaxing property and hence used in the management of musculoskeletal ailments. It is devoid of adverse effects like gastritis, renal compensation, and respiratory depression and therefore found to be safe in most patients. Though earlier studies have shown that flupirtine produces analgesia, ${ }^{4,5}$ there is dearth of research related to its ability in yielding postoperative analgesia, and hence, the present study was carried out to assess the efficacy of flupirtine and piroxicam in postoperative pain using visual analog scale (VAS) score, the total amount of rescue analgesic required, and patient's satisfaction score and to evaluate safety profile of the above drugs using WHO causality scale.

\section{Materials and methods}

A randomized, open-label, parallel group, comparative study was conducted by the departments of pharmacology and orthopedics on patients undergoing lower limb surgery in a tertiary care hospital from January 2015 to July 2016. All patients of either gender aged between 18 and 50 years undergoing either elective or emergency lower limb surgery with or without the requirement of implants and belonging to American Society of Anaesthesiologists (ASA) grade I and II were recruited. Patients with hepatic and renal impairment, hemorrhagic disorders, bronchial asthma, addictive disorders such as smoking, alcoholism, any other drugs of abuse, history of peptic ulcer, and gastrointestinal bleeding, pregnant and lactating women, and those with known hypersensitivity to the study medications were excluded from the study.

A pro forma containing detailed information of each patient was designed according to the study protocol. Ethical clearance was obtained from the institutional ethics committee affiliated to Sri Devaraj Urs Medical College. Patients undergoing elective or emergency lower limb surgery were recruited. After obtaining written informed consent, the patients were randomized using block randomization method. A block size of four was used and with the help of computer, random numbers for all the patients were generated, and the patients were assigned to two groups of 38 patients each. Baseline assessments included demographic details, clinical history, and examination. The patients were acquainted to the pain scores prior to start of the treatment with the study drugs and were requested to report any adverse events that they experienced during their stay in the hospital. Following surgery, both the groups received single dose of tramadol $100 \mathrm{mg}$ injection intravenously in the recovery room. Six hours after surgery, patients in group F received flupirtine $100 \mathrm{mg}$ twice daily and group P received piroxicam $20 \mathrm{mg}$ twice daily orally for 5 days.

Intensity of pain was measured using VAS score from 0 to 10 ( 0 is no pain and 10 is worst pain). VAS score was measured at baseline (before the study medication was administered) and at 2, 4, 8, 12, 24, 48, 72, 96, and 120 hours. Total pain relief $\left(\right.$ TOTPAR $_{24}$ ) score for first 24 hours was also assessed. The objective evaluation of pain was done using Behavioral Pain Assessment Scale (BPAS) (scored 0-10) and functional activity score (FAS) (scored A-C) at 12, 24, 48, 72, 96, and 120 hours.

Patient's pulse rate, blood pressure, and respiratory rate were monitored immediately after recovery from anesthesia and at 2, 4, 8, 12, 24, 48, 72, 96, and 120 hours postoperatively. Patients were administered tramadol $100 \mathrm{mg}$ injection intravenously as rescue medication if VAS score was $>3$ during the postoperative period. Patients' satisfaction with respect to pain relief was assessed using patient satisfaction score (PSS) at the end of 24 hours postoperatively. Adverse effects for both the drugs were monitored and causality assessed using WHO scale.

\section{Statistical methods}

To detect a mean difference of 0.7 in VAS with an effect size of 0.75 , an alpha error of $5 \%$, and a power of $80 \%$ with a dropout rate of $10 \%$, the required sample size was calculated to be 38 patients per group. ${ }^{5}$ The demographic data were assessed using descriptive statistics. The VAS and BPAS scores were assessed by repeated measures ANOVA followed by Bonferroni post hoc test within the group and unpaired $t$-test between the groups. TOTPAR ${ }_{24}$ and FAS were analyzed using descriptive statistics. PSS and need for rescue analgesia were analyzed using Mann-Whitney $U$ test and chi-squared test, respectively. Adverse effects were analyzed using Fischer's exact test. $p$-value $<0.05$ was considered to be statistically significant.

\section{Results}

A total of 76 patients undergoing lower limb surgeries were recruited in this study. They were randomized using 
computer-generated, block randomization method to either group F or P. The surgeries were performed under spinal anesthesia using $3 \mathrm{~mL}$ of $0.5 \%$ bupivacaine. All the patients received intravenous fentanyl $25 \mu \mathrm{g}$ intraoperatively, and following surgery, they received single dose of tramadol $100 \mathrm{mg}$ injection intravenously in the recovery room. Patients in group F received flupirtine $100 \mathrm{mg}$ capsule and those in group $\mathrm{P}$ received piroxicam $20 \mathrm{mg}$ tablet. Both the drugs were administered orally 6 hours after surgery and twice daily for the next 5 days. Seventy one patients completed the study. Figure 1 represents patient recruitment and follow-up.

The baseline characteristics were comparable in both the groups (Table 1). Among the 76 patients recruited, 65 were male and 11 female with an overall literacy rate of $59.21 \%$. The type of surgeries are shown in Table 1, those requiring the placement of orthopedic implants contributed to $46.1 \%$ of the total patients, and the distribution of type of surgeries between the groups was comparable ( $p=0.054)$.

Table 2 shows the reduction in VAS score over time in patients of both the groups. The reduction in intensity of pain in Groups F and $\mathrm{P}$ was statistically significant at 48 hours and onwards compared to baseline. Piroxicam significantly reduced pain compared to flupirtine at 8,12 , and 120 hours. The pain experienced by a patient was also graded as mild, moderate, and severe. Moderate pain was observed up to 72 hours by $55.9 \%$ patients in group $\mathrm{F}$ and $48.6 \%$ in group $\mathrm{P}$, thereafter the intensity reduced to mild pain in both the groups.

The area under the curve for postoperative pain in patients receiving two different medications (Figure 2) was calculated by trapezoid rule which showed that the pain intensity expressed as TOTPAR ${ }_{24}$ for the first 24 hours was lesser with piroxicam (116.38) than flupirtine (127.8), and a similar observation was noted there after upto 120 hours (piroxicam 332.64 and flupirtine 360).

A decrease in mean BPAS scores was observed in both the groups (Figure 3) and was statistically significant within the group at all-time intervals of assessment after 24 hours postoperatively, compared to baseline. At baseline, only $23 / 71$ of the patients could be assessed for FAS, of which $65.2 \%$ and $34.8 \%$ had mild to severe limitation of activity in groups $\mathrm{F}$ and $\mathrm{P}$, respectively (Table 3 ). In group F, 6\% of patients and, in group $\mathrm{P}, 27 \%$ had no limitation of activity at 72 hours, and this improvement was observed in 59\% and $54 \%$ of patients at 120 hours.

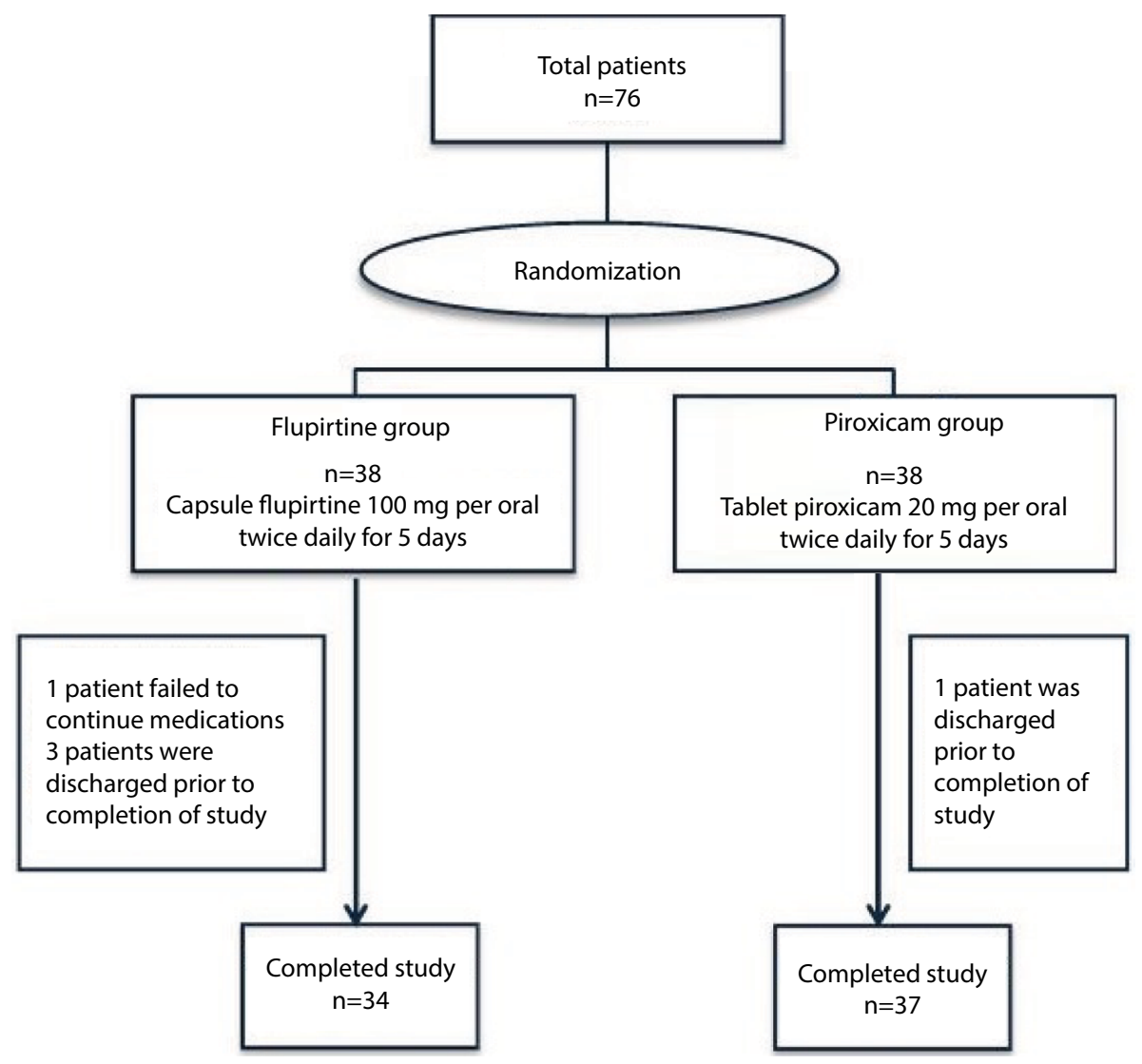

Figure I Flowchart representing recruitment of patients. 
Table I Baseline characteristics of all patients in both the groups

\begin{tabular}{llll}
\hline Variables & Flupirtine group $(\mathbf{n}=\mathbf{3 8})$ & Piroxicam group $(\mathbf{n}=\mathbf{3 8})$ & $\mathbf{p}$-value \\
\hline Male/female & $32 / 6$ & $33 / 5$ & 0.744 \\
Age, mean \pm SD (years) & $35.08 \pm 10.42$ & $35.08 \pm 10.22$ & 1.000 \\
Duration of surgery, mean \pm SD (hours) & $1.70 \pm 0.85$ & $1.48 \pm 0.86$ & 0.283 \\
Type of surgery & & & 0.054 \\
Implant placement surgeries & 21 & 14 & 14 \\
Implant removal & 5 & 10 & \\
Others & 12 & & \\
\hline
\end{tabular}

Table 2 VAS score in patients of both the groups at various time points

\begin{tabular}{|c|c|c|c|c|c|}
\hline \multirow{2}{*}{$\begin{array}{l}\text { Time in hours } \\
\text { postoperatively }\end{array}$} & \multicolumn{2}{|c|}{ Flupirtine group $(n=34)$} & \multicolumn{2}{|c|}{ Piroxicam group $(n=37)$} & \multirow[t]{2}{*}{$p$-value } \\
\hline & Mean \pm SD & $p$-value & Mean \pm SD & $p$-value & \\
\hline Baseline & $5.8 \pm 1.6$ & & $5.6 \pm 1.7$ & & 0.681 \\
\hline 2 & $5.4 \pm 1.2$ & 1.000 & $5.1 \pm 1.4$ & 0.660 & 0.253 \\
\hline 4 & $5.6 \pm 1.5$ & 1.000 & $5.3 \pm 1.6$ & 1.000 & 0.390 \\
\hline 8 & $5.8 \pm 1.3$ & 1.000 & $5.1 \pm 1.5$ & 1.000 & $0.028^{\#}$ \\
\hline 12 & $5.3 \pm 1.1$ & 1.000 & $4.5 \pm 1.6$ & 0.060 & $0.032^{\#}$ \\
\hline 24 & $4.8 \pm 1.3$ & 0.171 & $4.7 \pm 1.4$ & 0.058 & 0.713 \\
\hline 48 & $4.4 \pm 1.1$ & $0.006 *$ & $4.1 \pm 1.5$ & $0.0001 *$ & 0.354 \\
\hline 72 & $3.8 \pm 1.2$ & $0.000 I^{*}$ & $3.6 \pm 1.3$ & $0.0001 *$ & 0.502 \\
\hline 96 & $3.1 \pm 1.1$ & $0.000 I^{*}$ & $2.8 \pm 1.3$ & $0.0001 *$ & 0.346 \\
\hline 120 & $2.6 \pm 1.1$ & $0.0001 *$ & $2.1 \pm 0.8$ & $0.0001 *$ & $0.02 I^{\#}$ \\
\hline
\end{tabular}

Notes: *Comparison with baseline; "intergroup comparison.

Abbreviation: VAS, visual analog scale.

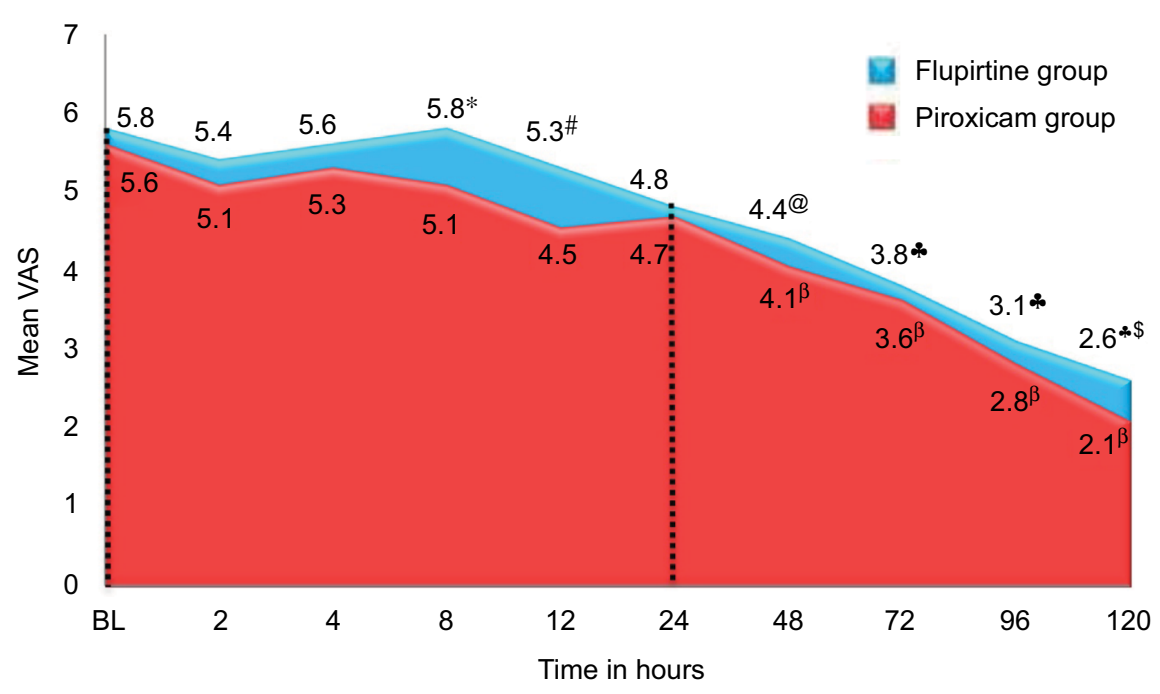

Figure 2 Areas under the curve for flupirtine and piroxicam.

Notes: ${ }^{\circledR} p=0.006,{ }^{*} p=0.000$ I, comparison with baseline in flupirtine group. ${ }^{\beta} p=0.000$ I, comparison with baseline in piroxicam group. Intergroup comparison; ${ }^{*} p=0.028$ at 8 hours, ${ }^{\#} p=0.032$ at 12 hours, ${ }^{\$} p=0.02 I$ at $I 20$ hours.

Abbreviations: BL, baseline; VAS, visual analog scale.

In $55.9 \%$ of patients in group $\mathrm{F}$ and $59.5 \%$ in Group P, the satisfaction score was "Good" (Figure 4), and it was comparable $(p=0.698)$. The rescue analgesic tramadol was required by 18 and 22 patients in groups $F$ and $P$, respectively, and it was insignificant between the groups $(p=0.580)$. Most patients $(62.5 \%)$ in both the groups required one to two doses of the medication over the study period of 5 days, and there was no significant difference in the number of doses between the groups $(p=0.365)$.

The most common adverse effect was dyspepsia which was observed in one patient receiving flupirtine and in three patients receiving piroxicam which was not statistically significant $(p=0.40)$. The effects of the study drugs on pulse, blood pressure, and respiratory rate were assessed at different 


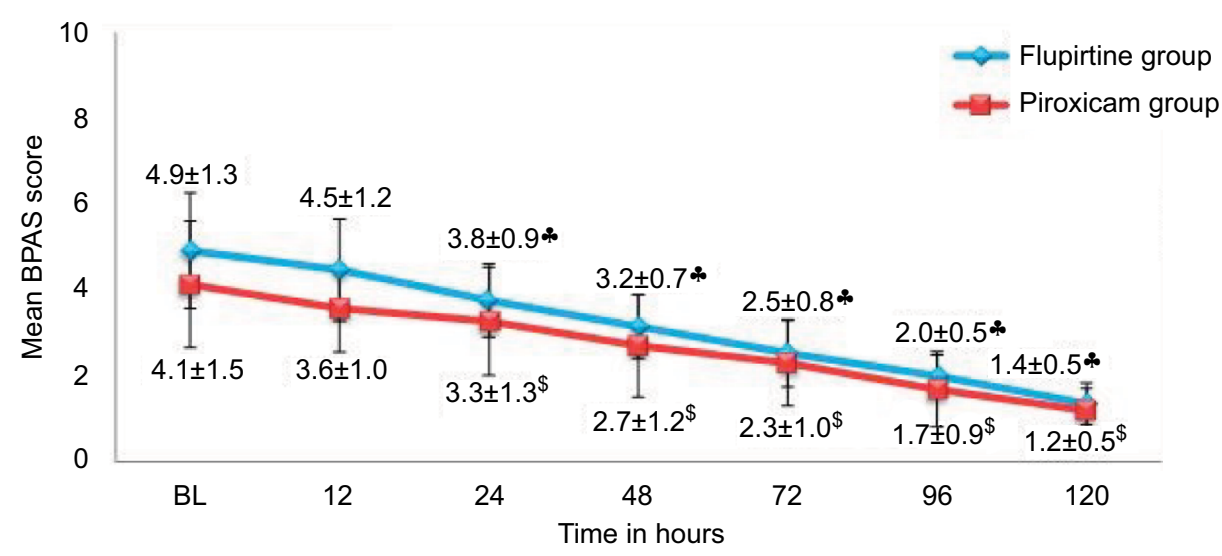

Figure 3 Comparison of mean BPAS scores within the groups.

Notes: ${ }^{*} p=0.000$ I, comparison with baseline in flupirtine group. ${ }^{\$} p=0.0001$, comparsion with baseline in piroxicam group.

Abbreviations: BL, baseline; BPAS, behavioral pain assessment scale.

Table 3 Comparison of functional activity scores between two groups

\begin{tabular}{llllll}
\hline $\begin{array}{l}\text { Time interval } \\
\text { (hours) }\end{array}$ & Group & A (\%) & B (\%) & C (\%) & NA (\%) \\
\hline Baseline & Flupirtine & 0 & $5(14.7)$ & $10(29.4)$ & $19(55.9)$ \\
& Piroxicam & 0 & $5(13.5)$ & $3(8.1)$ & $29(78.4)$ \\
12 & Flupirtine & 0 & $16(47.1)$ & $18(52.9)$ & 0 \\
& Piroxicam & $1(2.7)$ & $19(51.4)$ & $17(45.9)$ & 0 \\
24 & Flupirtine & 0 & $24(70.6)$ & $10(29.4)$ & 0 \\
48 & Piroxicam & $3(8.1)$ & $23(62.2)$ & $11(29.7)$ & 0 \\
72 & Flupirtine & 0 & $32(94.1)$ & $2(5.9)$ & 0 \\
& Piroxicam & $5(13.5)$ & $28(75.7)$ & $4(10.8)$ & 0 \\
96 & Flupirtine & $2(5.9)$ & $31(91.2)$ & $1(2.9)$ & 0 \\
120 & Piroxicam & $10(27.0)$ & $27(73.0)$ & 0 & 0 \\
& Flupirtine & $11(32.4)$ & $22(64.7)$ & $1(2.9)$ & 0 \\
& Piroxicam & $16(43.2)$ & $21(56.8)$ & 0 & 0 \\
\hline
\end{tabular}

Notes: A: no limitation (perform task without pain). B: mild limitation (perform task with minimal pain). C: severe limitation (unable to perform the task). NA: could not assess.

time intervals. There was no significant difference in these vital parameters within or between the groups.

\section{Discussion}

Pain management in postsurgical patients has been a major concern over the last few decades. ${ }^{6}$ It is one of the important aspects to be considered because it is closely related to good clinical outcome. Orthopedic procedures can cause severe intraoperative and postoperative pain which is indicative of tissue damage and is usually a major clinical problem occurring in $62 \%-65 \%$ of patients. ${ }^{7,8}$ Thus, effective pain management is needed for the recovery of range of movements, muscle strength for ambulation, rapid rehabilitation, shorter hospital stay, and to improve the quality of life of patients. ${ }^{9}$

In our study, majority were male patients with a mean age of $35 \pm 10$ years. Only patients undergoing lower limb surgeries were recruited as the sensitivity of pain differs among different bones. The distribution of type of surgeries between the groups was comparable and implant placements were predominant. The mean duration of surgery was similar in patients receiving either medication. At baseline, the mean VAS score was comparable between the two groups. The reduction in pain was significant at all points of time following 48 hours treatment compared to baseline in both the groups. This observation partly correlates with the findings of another study, ${ }^{10}$ indicating that though both the study drugs have a slight delay in onset, they have a longer duration of analgesia. Flupirtine used as preemptive analgesic in laparoscopic cholecystectomy surgery had provided adequate pain relief postoperatively in a study conducted by Yadav et $\mathrm{al}^{11}$ and in postabdominal hysterectomy study conducted by Thapa et al. ${ }^{12}$ These studies indicate that preemptive use of flupirtine could provide immediate postoperative pain relief; hence, administering this drug preemptively followed by postoperative dosing may be a better method for postoperative analgesia in orthopedic surgeries. 


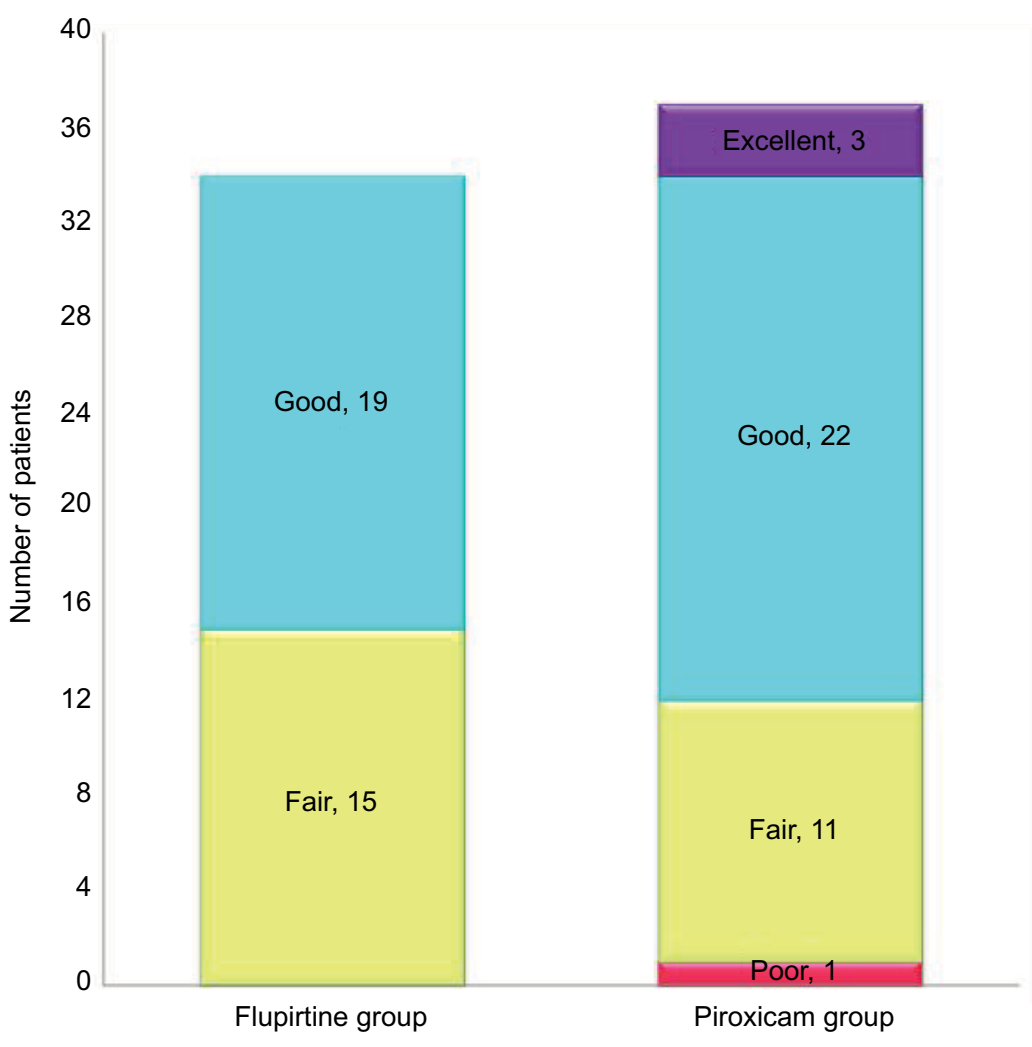

Figure 4 Patient satisfaction score in both the groups at the end of 24 hours.

In patients who received piroxicam, reduction in pain was significant at 8,12 , and 120 hours postoperatively compared to flupirtine. This suggests that patients receiving piroxicam had better analgesic effect compared to flupirtine. The TOTPAR 24 which is a measure of continuous pain relief also showed that piroxicam was better in relieving pain. However, a study conducted by Yadav et al proved flupirtine to provide better analgesia than diclofenac ${ }^{13}$ and that by Naser et $\mathrm{al}^{5}$ showed equal efficacy of flupirtine and ibuprofen in terms of analgesia when used in gynecological surgeries. Similar studies by Attri et al and Ahuja et al, which compared flupirtine with other NSAIDs, also revealed no significant difference between the medications in reducing postoperative pain. ${ }^{5,10,14}$ These studies were conducted on patients undergoing gynecological and other abdominal surgeries unlike the current study which was carried out in limb surgeries. The extensiveness and increased sensitivity of osteoid tissues tend to result in severe postoperative pain following surgeries involving the bone. Thereby, the current study that assessed the efficacy of drugs on pain showed piroxicam to provide better analgesia than flupirtine.

The objective BPAS showed a reduction with both the medications, which reflects pain relief, but another study has shown reduction in VAS score at an earlier point of time compared to BPAS score. ${ }^{15}$ Improvement in the FAS reflects improvement in the range of movements in the operated limb. Majority of patients who had moderate to severe limitation of activity at baseline improved over the first 96 hours, and this was earlier in patients receiving piroxicam.

PSS at the end of 24 hours was good in 56\% and $60 \%$ in flupirtine and piroxicam groups. There was no significant difference in their satisfaction score, which indicates that patients were contended with the medication they received. However, a study comparing similar drugs showed flupirtine to have superior satisfaction score. ${ }^{14}$ In our study, the requirement of rescue analgesic was similar with both the medications, which was similar to another study. ${ }^{14}$ In this study, the adverse effects were dyspepsia and dizziness. Dyspepsia was the only adverse effect in piroxicam group and is due to inhibition of protective effect of prostaglandin on gastric mucosa. Dizziness that occurred in a patient who received flupirtine could be attributed to the hypotension, which is a common side effect with flupirtine due to its effect on the $\mathrm{K}_{\mathrm{v}} 7$ channels in vascular smooth muscle. ${ }^{4}$ The study drugs showed no effect on the vital parameters such as pulse rate, blood pressure, and respiratory rate.

The findings of our study imply that pain relief was similar with both the drugs but the onset was earlier with piroxicam. In individuals with history or risk of dyspepsia, flupirtine may be preferred. 


\section{Conclusion}

Flupirtine and piroxicam reduced pain effectively in patients following lower limb surgery but onset of pain relief was earlier with piroxicam.

\section{Acknowledgments}

The abstract of this paper was presented as an oral presentation with complete findings at the World Congress of Gerontology and Geriatrics, 4th International Conference, Healthy Ageing in the Changing World 2016; Bengaluru, India; November 24, 2016. The oral presentation was published in "Abstract Book" in An International Convention for Medical and Technological Advancements in Healthcare, by Bio Genesis Health Cluster; $h$ ttp://geriatricsconference.com/ oral-presentations/.

\section{Disclosure}

The authors report no conflicts of interest in this work.

\section{References}

1. Cardiff University. Acute postoperative pain: definition of acute pain. Available at: http://www.paincommunitycentre.org/article/acutepostoperative-pain-definition-acute-pain. Accessed October 10, 2014.

2. Imani F, Rahimzadeh P. Gabapentinoids: gabapentin and pregabalin for postoperative pain management. Anesth Pain. 2012;2:52-53.

3. Wu S, Hsu M, Liao Y, Wu F, Jong Y, Lo Y. Evidence for inhibitory effects of flupirtine, a centrally acting analgesic, on delayed rectifier $\mathrm{K}+$ currents in motor neuron-like cells. Evid Based Complement Alternat Med. 2012;2012:Article ID 148403.
4. Szelenyi I. Flupirtine, a re-discovered drug, revisited. Inflamm Res. 2013;62:251-258.

5. Naser SM, Sarkar N, Biswas A, et al. Efficacy and safety of flupirtine maleate and tramadol hydrochloride in postoperative pain managementa prospective randomized double blinded study. J Indian Med Assoc. 2012;110:158-160.

6. Chandrakantan A, Glass PS. Multimodal therapies for postoperative nausea and vomiting, and pain. Br J Anaesth. 2011;107(Suppl 1):i27-i40.

7. Srivastava M, Singh M, Kapoor D. Pain management in orthopaedic surgeries: a major concern. Punjab J Orthop. 2012;13:30-39.

8. Layzell M. Current interventions and approaches to postoperative pain management. Br J Nurs. 2008;17:414-419.

9. Salmon P, Hall GH, Peerbhoy D, Shenkin A, Parker C. Recovery from hip and knee arthroplasty: patient's perspective on pain, function, quality of life and well-being up to 6 months postoperatively. Ach Phys Med Rehabil. 2000;82:360-366.

10. Attri JP, Sandhu GK, Khichy S, Singh H, Singh K, Sharan R. Comparative evaluation of oral flupirtine and oral diclofenac sodium for analgesia and adverse effects in elective abdominal surgeries. Anesth Essays Res. 2015;9:72-78.

11. Yadav G, Behera SS, Das SK, Jain G, Choupoo S, Raj J. Role of flupirtine as a preemptive analgesic in patients undergoing laparoscopic cholecystectomy. J Anaesthesiol Clin Pharmacol. 2015;31:169-173.

12. Thapa D, Ahuja V, Dass C, Gombar S, Huria A. Effect of preoperative flupirtine on postoperative morphine sparing in patients undergoing total abdominal hysterectomy. Saudi J Anaesth. 2016;10:58-63.

13. Yadav G, Choupoo S, Das SK, et al. Evaluating the role of flupirtine for postcraniotomy pain and compare it with diclofenac sodium: a prospective, randomized, double blind, placebo-controlled study. J Neurosurg Anesthesiol. 2014;26:32-36.

14. Ahuja V, Mitra S, Kazal S, Huria A. Comparison of analgesic efficacy of flupirtine maleate and ibuprofen in gynaecological ambulatory surgeries: a randomized controlled trial. Indian J Anaesth. 2015;59: 411-415.

15. Ahlers SJ, van der Veen AM, van Dijk M, Tibboel D, Knibbe CA. The use of the behavioral pain scale to assess pain in conscious sedated patients. Anesth Analg. 2010;110:127-133.
Journal of Pain Research

\section{Publish your work in this journal}

The Journal of Pain Research is an international, peer reviewed, open access, online journal that welcomes laboratory and clinical findings in the fields of pain research and the prevention and management of pain. Original research, reviews, symposium reports, hypothesis formation and commentaries are all considered for publication.

\section{Dovepress}

The manuscript management system is completely online and includes a very quick and fair peer-review system, which is all easy to use. Visit http://www.dovepress.com/testimonials.php to read real quotes from published authors. 